\title{
Structures and Structural Information ${ }^{\dagger}$
}

\author{
Mark Burgin 1,* and Rainer Feistel ${ }^{2}$ \\ 1 Department of Computer Science, University of California, 520 Portola Plaza, Los Angeles, CA 90095, USA \\ 2 Leibniz Institute for Baltic Sea Research, Warnemünde D-18119, Germany; \\ rainer.feistel@io-warnemuende.de \\ * Correspondence: mburgin@math.ucla.edu \\ + Presented at the IS4SI 2017 Summit DIGITALISATION FOR A SUSTAINABLE SOCIETY, Gothenburg, \\ Sweden, 12-16 June 2017.
}

Published: 9 June 2017

\begin{abstract}
Structures play a crucial role in the world. They define things and thoughts, processes and functions, images and ideas. The goal of this research is to study structural information, which is intrinsically connected to structures. Here we present two approaches. In one of them, structural information is information in structures. In the other one, structural information is information about structures.
\end{abstract}

Keywords: information; structure; structural information; interaction; correctness; knowledge

\section{Introduction}

Everything has a structure and this structure makes things such as they are. This was declared by Aristotle for material things and demonstrated in the general theory of structures developed in $[1,2]$ for the whole generality of existing and possible objects. This is the core reason of importance of structural information, which provides and/or changes knowledge about structures.

As Brinkley writes, "implicit in the word "structure" is not only the concept of elementary units or parts, but also the interdependence and relationships of those parts to form a whole and it can thus be argued that modern science has adopted a structural approach to understanding the natural world, in which parts are defined and the interactions among them are explored" [3,4].

Structural information is the core of structuralism, the heart of structural realism and the basic essence of structural informatics.

The goal of this work is to study structural information based on the general theory of information [1,5-8], research of Feistel and Ebeling [9-12] and works of other authors in this area. The goal is developing more comprehensive and advanced knowledge about structural information and its relation to symbolic information.

\section{Structural Information in the Context of the General Theory of Information}

It is possible to comprehend structural information in different ways. For instance, Bates [13] treats structural information as "the pattern of organization of matter and energy," while Reading [14] defines it as "the way the various particles, atoms, molecules, and objects in the universe are organized and arranged."

At first, we consider the approach developed in the general theory of information. The general theory of information discerns information in the broad sense (Ontological Principle O2) and information in the strict sense (Ontological Principle O2a).

Structural information is a kind of information in the strict sense being defined as a capacity to change the subsystem of knowledge in an intelligent system. 
It is possible to differentiate three types of structural information.

1. Inherent structural information is information in structures.

2. Descriptive structural information is information about structures.

3. Constructive structural information is information that allows building knowledge about structures.

These definitions allow getting key properties of structural information. Let us consider some of them.

1. Structural information can be more correct or less correct.

Correctness of structural information about a system depends on correctness of knowledge produced by this information [7]. As we know, some knowledge can be more correct, better representing the essence of the system, while other knowledge is less correct, providing a worse representation of the fundamental nature of the system.

Here are two examples.

Example 1. For a long time, people believed that the Earth was flat, i.e., had the structure of a plane. Then scientists found that the Earth had the structure of a ball. Then scientists assume that the Earth had the structure of a geoid.

Example 2. For a long time, people believed that in the structure of the Solar system, the Sun rotated around the Earth.Then scientists found that the Earth rotated around the Sun and the orbit had the structure of a circle.Then scientists assume that the Earth rotates around the Sun and the orbit had the structure of an ellipse.

2. As a rule, structural information about a system is not unique.

Many researchers believe that each (e.g., a natural) system has a unique structure. At the same time, according to the general theory of structures [1], any system has several structures. For instance, the structure of a table on the level of its parts is essentially different from the structure of this table on the level of molecules as well as from the structure of this table on the level of its parts such as legs. In essence, material systems, which people can see with their eyes and touch with their hands, have structural information on different levels.

3. Structural information about a system can be inherent to this system, inbuilt in the interaction with the system or innate for an image of the system.

Indeed, as it is stated above, structure makes things such as they are. Naturally, structural information reflects this identity of things although structural information about different systems and objects can be similar.

4. Processes in a system can change structural information about this system.

Indeed, the evolution (development) of a system can produce an essentially new structure when the system is changed, even becoming another system. For instance, butterflies have the four-stage life cycle. In it, winged adults lay eggs, which later become caterpillars, which later pupate in a chrysalis, while at the end of the metamorphosis, the pupal skin splits and a butterfly flies off.

5. Structural information about a system describes this system to a definite extent of precision, i.e., structural information can be more precise and less precise.

For instance, the Copernican model (structure) of the Solar System is more precise than the Ptolemaic model (structure) of the Solar System. Another example comes from mathematics where mathematicians are striving to find the decimal structure of the number $\pi$ with higher and higher precision. 
6. For complex systems, it is possible to consider structural information on different levels and various scales.

For instance, it is possible to treat the structure of a human being on the level of visible parts, on the level of its functional systems, on the level of inner organs, on the level of cells, on the level of chemical compounds or on the level of molecules.

7. Structural information about a subsystem of a system is not always a part of the structural information about this system.

For instance, when we consider an organism as a system of its visible parts, the structure of its nervous system is not a part of this structure.

8. The process of conversion of structural information about a system into knowledge about this system is, in essence, structuration of this system.

Indeed, people get information about different objects in the form of raw data. Only after reception of this information, the brain converts these data into knowledge and this knowledge is often about the structure of studied objects. It is natural to treat data as symbolic representation of structural information, where correct structural information may be represented incorrectly by symbolic information. This may happen in the measuring device, or in the transmission or storage, or in the brain, etc.

Note that the general theory of information provides other possibilities for defining structural information. For instance, it can be information that changes the system of beliefs of an intelligent system.

\section{Structural Information as the Intrinsic Property}

At the same time, Feistel and Ebeling suggest the vision of structural information, in which structural information may no longer be restricted to changing just "knowledge in an intelligent system", and may more generally be defined as the capacity of a physical system, the "carrier of structural information", to cause changes in a second physical system, the "receiver of structural information" [9-12].

If in particular, the receiver is the same system as the carrier but at some later point of time, reversible microscopic dynamics described by the Liouville equation is universally understood as "conserving [microscopic] [structural] information" [15-17]. In contrast to this, irreversible macroscopic dynamics is commonly associated with a loss of [macroscopic] [structural] information, directly related to the growth of thermodynamic entropy $[9,10]$. In the sense of Planck [18] who wrote that "a macroscopic state always comprises a large number of microscopic states that combine to an average value", macroscopic structural information represents a portion of the microscopic structural information of a given system. This approach is consistent with the approach from Section 2. Namely, a system may have different structures, such as a microscopic structure and a macroscopic structure. In each of those structures, there exists related structural information. While the microscopic information may be conserved, the macroscopic information may decay according to the 2nd law of thermodynamics. That is, the different structures of the same system may possess different symmetries and may follow different laws. Irreducibilities of this kind indicate emergent properties or structures [10].

In his famous article "The RNA world" about the origin of life, Gilbert [19] wrote about "the useful distinction between information and function", that "information storage needs to be one-dimensional, for ease of copying, but molecules with enzymatic functions tend to be tight three-dimensional structures". This quotation demonstrates that the term "information" is in common scientific use in situations in which "knowledge in an intelligent system" does not exist at all, such as before life emerged on Earth. Gilbert refers to the structural information given by the sequence of building blocks in a chain molecule. This information is received later by the same molecule when it folds into a functioning enzyme, and by other reactants then catalyzed by it. Similarly, the information scientist Klaus Fuchs-Kittowski [20] speaks about "information" that was 
generated in evolution for the first time when life began, and has allowed living structures to survive to the present day. Without retaining this structural information, living beings would be as astronomically rare among random chemical mixtures [21] as are sensible books among the random texts in Jorge Luis Borges' "Library of Babel" [22].

Structural information available from a carrier depends on the receiver determining what portion of this information is actually received. If, for example, the receiver is a thermometer and the carrier is liquid, then all information received is the temperature of the liquid. Structural information can be extracted from a given system by "measurement" when e.g., a sensor is used as a receiver. Structural information can be quantified if it is comparable to the structural information of a reference system, such as the length scale of a mercury thermometer.

A numerical value being the result of a comparison between the same kinds of structural information available from two different systems, such as by counting their parts, is a "measurement result". Numbers represent information in the symbolic form, or as "symbolic information". The meaning of symbolic information is subject to convention (such as what "reference" system is used) and is no longer a portion of the structural information of the carrier, such as printed symbols on a sheet of paper. Very different structural information carriers can carry the same symbolic information. Symbolic information is restricted to the realm of life [9-11], such as in the form of genetic DNA molecules or human knowledge, and emerged from structural information in the course of evolution by a transition process regarded as ritualisation.

The origin of life is a famous example for the ritualisation transition from structural to symbolic information. Organic chain molecules, see the quotation of Gilbert [19] above, carried structural information in the form of certain sequences of their elements. Some of those resulted in catalytic properties that supported the copying of the chain molecule, such as in a RNA-replicase cycle $[19,23,24]$. In the course of evolution, the initial chain molecules developed to carriers of symbolic information in a complex multi-step process $[9,23]$. Similar features of the ritaulisation transition are observed, for example, when humans began to speak, or when digital computers replaced mechanical calculators.

The codons of the modern genetic code are the symbols of genetic information, and the phenotypic traits of the developed organism represent the meaning of that information. The symbols also carry structural information, but due to the arbitrariness of symbols in a mature coding system, this structural information is irrelevant for the meaning they express. The properties of the ink used by Mozart for writing down his music are irrelevant for the beauty of its acoustic performance [25].

The main distinction between structural and symbolic information is that the meaning of structural information is inseparably bound to its physical structure, while symbolic information is free insofar as arbitrary symbols may take over the same role in the information processing system. Symbolic information possesses the code symmetry, or coding invariance, that emerges during the ritualisation transition [26,27]. Related to this symmetry is the structural information of symbols that preserves traces of the evolution of the symbolic information-processing system. So, it is known from many spoken words that they have onomatopoetic roots. The similarity of words used in the various languages provides structural information about the migration histories of the populations. In the same way, the physical and chemical properties of the genetic apparatus carry structural information about the beginning of life in an ancient past.

\section{Conclusions}

Presented here approaches to structural information reflect the basic ontological problem of the role of an observer. Namely, the question is whether properties of physical things exist by themselves or their existence is dependent on the presence of an observer. In Section 2 of this work, structural information is treated from the point of view of an observer although such an observer can be not only a human being but any cognitive system. In Section 3 of this work, structural information is regarded as an intrinsic property of physical things independent of any observer. 
Conflicts of Interest: The authors declare no conflict of interest.

\section{References}

1. Burgin, M. Structural Reality; Nova Science Publishers: New York, NY, USA, 2012.

2. Burgin, M. Theory of Knowledge: Structures and Processes; World Scientific: New York, NY, USA; London, UK; Singapore, 2016.

3. Brinkley, J.F. Structural informatics and its applications in medicine and biology. Acad. Med. 1991, 66, 589-591.

4. Brinkley, J.F. What Is Structural Informatics? 1999. Available online: http://sig.biostr.washington.edu/ overview/si.html (accessed on 4 July 2017)

5. Burgin, M. Information: Problems, Paradoxes, and Solutions. Triple C 2003, 1, 53-70.

6. Burgin, M. Data, Information, and Knowledge. Information 2004, 7, 47-57.

7. Burgin, M. Theory of Information: Fundamentality, Diversity and Unification; World Scientific: New York, NY, USA; London, UK; Singapore, 2010

8. Burgin, M. Information Dynamics in a Categorical Setting. In Information and Computation; World Scientific: New York, NY, USA; London, UK; Singapore, 2011; pp. 35-78

9. Feistel, R.; Ebeling, W. Physics of Self-Organization and Evolution; Wiley-VCH: Weinheim, Germany, 2011.

10. Feistel, R.; Ebeling, W. Entropy and the Self-Organization of Information and Value. Entropy 2016, $18,193$.

11. Ebeling, W.; Feistel, R. Selforganization of Symbols and Information. In Chaos, Information Processing and Paradoxical Games: The Legacy of John S. Nicolis; Nicolis, G., Basios, V., Eds.; World Scientific Pub Co.: Singapore, 2015; pp. 141-184

12. Feistel, R. Self-organisation of Symbolic Information. Eur. Phys. J. Spec. Top. 2016, doi:10.1140/epjst/e2016-60170-9.

13. Bates, M.J. Information and knowledge: An evolutionary framework for information science. Inform. Res. 2005, 10, 239.

14. Reading, A. The Biological Nature of Meaningful Information. Biol. Theory 2006, 1, $243-249$.

15. Hawking, S. The Universe in a Nutshell; Bantam Books: New York, NY, USA, 2001

16. Zhang, B.; Cai, Q.-Y.; Zhan, M.-S.; You, L. Information conservation is fundamental: Recovering the lost information in Hawking radiation. Int. J. Mod. Phys. D 2013, 22, 1341014.

17. Ginnings, S.B. Black holes, quantum information, and the foundations of physics. Phys. Today 2013, 66, 30-35.

18. Planck, M. Theorie der Wärmestrahlung, 6. Auflage; Johann Ambrosius Barth: Leipzig, Germany, 1966

19. Gilbert, W. The RNA world. Nature 1986, 319, 618.

20. Fuchs-Kittowski, K. Information und Biologie-Informationsentstehung als neue Kategorie für eine Theorie der Biologie. In Biochemie-ein Katalysator der Biowissenschaften; Kolloquium der Leibniz-Sozietät am 20. Nov. 1997 zum 85. Geburtstag von Samuel Mitja Rapoport, Sitzungsberichte der Leibniz-Sozietät, Band 22; trafo-Verlag: Berlin,Germany, 1999.

21. Monod, J. Chance and Necessity; Alfred A. Knopf: New York, NY, USA, 1971.

22. Borges, J.L. The Library of Babel; Penguin: New York, NY, USA, 1998.

23. Ebeling, W.; Feistel, R. On the Evolution of Biological Macromolecules. I. Physico-Chemical Self-Organization. Stud. Biophys. 1979, 75, 131-146.

24. Eigen, M. From Strange Simplicity to Complex Familiarity; Oxford University Press: Oxford, UK, 2013.

25. Eigen, M. Stufen zum Leben; Piper: München,Germany, 1992.

26. Feistel, R. Ritualisation und die Selbstorganisation der Information. In Selbstorganisation und Determination; Niedersen, U., Pohlmann, L., Eds.; Duncker \& Humblot: Berlin, Germany, 1990; pp. 83-98.

27. Feistel, R. Emergence of Symbolic Information by the Ritualisation Transition. In Information Studies and the Quest for Transdisciplinarity; Burgin, M., Hofkirchner, W., Eds.; World Scientific: Singapore, 2017; pp. 115-164.

(C)2017 by the authors. Licensee MDPI, Basel, Switzerland. This article is an open accessarticle distributed under the terms and conditions of the Creative Commons Attribution(CC BY) license (http://creativecommons.org/licenses/by/4.0/). 